\title{
GMR
}

\section{G-395A polymorphism in the promoter region of the KLOTHO gene and hypertension among elderly (90 years and older) Chinese individuals}

L.L. Gao, X. Ding, D.M. Xie, M. Yang and B.R. Dong

The Center of Gerontology and Geriatrics, West China Hospital, Sichuan University, Chengdu, Sichuan, China

Corresponding author: M. Yang

E-mail: yangmier@gmail.com

Genet. Mol. Res. 14 (4): 15444-15452 (2015)

Received June 10, 2015

Accepted September 30, 2015

Published November 30, 2015

DOI http://dx.doi.org/10.4238/2015.November.30.22

ABSTRACT. The aim of this study was to examine the possible associations between the KLOTHO G-395A gene polymorphism and hypertension in Chinese nonagenarians and centenarians. The G-395A (rs1207568) in the promoter region of the KLOTHO gene was genotyped using a standard TaqMan allelic discrimination assay. We included 710 participants aged $93.5 \pm 3.2$ years in the analyses. The expression of the A allele of the KLOTHO G-395A polymorphism was significantly downregulated in the hypertension group compared to the control group (0.137 vs 0.200 , P < 0.001). The genotype distribution of the G-395A polymorphism between the hypertension and control groups was significantly different in women and smokers, and not in men or non-smokers. The mean systolic blood pressure, percentage of hypertension, and percentage of isolated systolic hypertension was significantly higher in the group with the GG genotype than in the group with the GA+AA genotype. Subjects expressing the $\mathrm{GA}+\mathrm{AA}$ genotype demonstrated a significantly lower risk of hypertension even after adjusting for age, gender, and other relevant risk factors compared to the population expressing the GG genotype (odds ratio, $0.68 ; 95 \%$ confidence interval: 0.49 to 0.95$)$. The $-395 \mathrm{~A}$ allele of the 
KLOTHO gene may be a protective genetic factor for hypertension in the Chinese population.

Key words: KLOTHO gene; Hypertension; Single nucleotide polymorphism

\section{INTRODUCTION}

Hypertension, one of the most common causes of preventable death, is known to increase with advancing age (Zeglin et al., 2009; Virdis et al., 2011). Hypertension is known to be the result of interactions between genetic and environmental factors (Kunes and Zicha, 2009). Current evidence indicates that genetic components contribute approximately $30 \%$ to $50 \%$ to the etiology of hypertension (El Shamieh et al., 2012). Several recent studies have suggested that single nucleotide polymorphisms (SNPs) in some candidate genes may be related to hypertension (Delles et al., 2010).

Discovered by Kuro-o et al. (1995) during the development of hypertensive transgenic mice models, the KLOTHO gene, a new anti-aging gene (Wang and Sun, 2009), has recently attracted considerable attention from researchers around the world (Kuro-o, 2009). The identification of SNPs in the human $K L O T H O$ gene is currently one of the most active areas of research. So far, more than 10 SNPs have been identified in the human KLOTHO gene, and linked to fasting glucose (Rhee et al., 2006b), coronary artery disease (Imamura et al., 2006; Rhee et al., 2006a), stroke (Kim et al., 2006), bone mineral density (Kawano et al., 2002), and kidney stone (Telci et al., 2011).

Among these, the G-395A polymorphism in the promoter region of the KLOTHO gene has been associated with blood pressure in several populations (Rhee et al., 2006b; Wang et al., 2010). For example, Rhee et al. (2006b) observed significantlyhigher systolic blood pressure levels in the A allele carriers of the G-395A polymorphism in Korean women. A more recentreport has suggested that the G-395A polymorphism might be associated with essential hypertension in the Chinese Han population (Wang et al., 2010). However, Shimoyama et al. (2009) reported the absence of any association between the mean systolic blood pressure (SBP) and the G-395A polymorphism in healthy Japanese people.

In 2005, we conducted a cross-sectional study of 870 elderly Chinese adults aged 90 years and older (Yue et al., 2010; Zhou et al., 2012), and built a relevant DNA specimen database. Most of the participants lived in their homeland for their entire lifetime and were never exposed to immigrants. The prevalence of hypertension was found to be extremely high (more than $60 \%$ ) in this population; therefore, this population presented a unique opportunity to study the genetic factors of hypertension. Therefore, this study was performed to examine the possible associations between the KLOTHO G-395A SNP and hypertension in Chinese nonagenarians and centenarians.

\section{MATERIAL AND METHODS}

\section{Study population}

Data and DNA samples used in this study were from obtained from the Project of Longevity and Aging in Dujiangyan (PLAD), a previously reported cross-sectional study (Yue et al., 2010; Zhou et al., 2012). The PLAD study was conducted in the rural area of Dujiangyan, a small town in southwestern China, during April 2005. A total of 1115 adults aged 90 years and older dwelling 
in this area were screened, among who, 870 subjects agreed to participate in this study. Trained interviewers visited all study participants at their homes or at community centers, and data was collected by conducting face-to-face interviews. Anthropometric measurements were performed and blood was collected by trained staff. The study protocol was approved by the Research Ethics Committee of Sichuan University. Informed consent was obtained from all of participants or their legal proxies.

\section{Genotyping of polymorphisms in the KLOTHO gene}

Genomic DNA was isolated from the whole blood samples drawn from the antecubital vein, using the TIANamp Blood DNA Midi Kit (DP332; Qiagen, Chatsworth, CA, USA) using standard procedures. The G-395A (rs1207568) polymorphism in the promoter region of the KLOTHO gene was genotyped using the TaqMan allelic discrimination assay, as described previously by Wang et al. (2010), using the following primers and probes: forward primer, 5'-TAGGGCCCGGCAGGAT-3'; reverse primer, 5'-CCTGGAGCGGCTTCGTC-3'; probe A 5'-(FAM) CCCCAAGTCGGGAAAAGTTG GTC(TAMRA)-3'; probe G 5'-(HEX) CCCCCAAGTCGGGGAAAGTTGGTC(TAMRA)-3' (TaKaRa Bio Inc., Dalian, China). The PCR was conducted in a $20 \mu \mathrm{L}$ reaction volume containing $10 \mu \mathrm{L}$ Premix Ex Taq, $1.5 \mu \mathrm{L}$ of each primer, $0.5 \mu \mathrm{L}$ probe $\mathrm{A}, 1 \mu \mathrm{L}$ probe $\mathrm{G}, 1 \mu \mathrm{L}$ of genomic DNA, and $4.25 \mu \mathrm{L}$ sterile double distilled water. The reaction conditions were as follows: an initial denaturation step at $95^{\circ} \mathrm{C}$ for $30 \mathrm{~s}$, followed by 40 cycles of denaturation at $95^{\circ} \mathrm{C}$ for $5 \mathrm{~s}$ and annealing for $30 \mathrm{~s}$ at $60^{\circ} \mathrm{C}$. The PCR was performed and analyzed on a Thermal Cycler Dice Real Time System (TaKaRa Bio Inc.).

The genotypes of the rs 1207568 polymorphism in the promoter of the KLOTHO gene were analyzed by random selection of $10 \%$ of the samples for forward and reverse sequencing. The results of the sequencing were identical to the results of the TaqMan allelic discrimination assay.

\section{Diagnosis of hypertension}

Qualified nurses or physicians measured the sitting blood pressure (BP) of all patients twice (interval of $10 \mathrm{~min}$ ) to the nearest of $2 \mathrm{mmHg}$ using a standard mercury sphygmomanometer (phase I and $\mathrm{V}$ of korotkoff sounds). The mean value of the two measurements was recorded. In this study, hypertension was defined according to the JNC VII criteria, as a systolic BP (SBP) of $\geq 140 \mathrm{mmHg}$ and/or a diastolic BP (DBP) of $\geq 90 \mathrm{mmHg}$ (Chobanian et al., 2003). Participants who met either of the following criteria were considered to have hypertension: the measured BP (during the interview) met the JNC VII criteria mentioned above,or a confirmed clinical diagnosis of hypertension based on formal medical records, and currently receiving anti-hypertension medication. In addition, isolated systolic hypertension (ISH) was defined as a SBP $\geq 140 \mathrm{mmHg}$ and a DBP $<90 \mathrm{mmHg}$, based on the measured BP during the interview.

\section{Measurement of covariates}

The height and weight of all patients was measured using a wall-mounted stadiometer and a digital floor scale to the nearest $0.1 \mathrm{~cm}$ and $0.1 \mathrm{~kg}$, respectively. Body mass index (BMI) was calculated by dividing the body weight in kilograms by the height in squared meters $\left(\mathrm{kg} / \mathrm{m}^{2}\right)$. Laboratory analyses included the measurement of fasting plasma glucose (FPG), postprandial blood glucose (PBG), total cholesterol (TC), triglyceride content (TG), high density lipoprotein 
cholesterol (HDL-C), and low density lipoprotein cholesterol (LDL-C). Diabetes was diagnosed based on the criteria of the American Diabetes Association (ECDCDM, 2003), or if the subjects had a history of diabetes and were currently using antidiabetic agents. In addition, the following baseline information was included in the analyses: age, gender, and cigarette smoking status (smoker, defined ashaving smoked $\geq 100$ cigarettes in his/her lifetime (Ryan et al., 2012), or nonsmoker).

\section{Statistical analysis}

The categorical variables are presented as percentages (\%), whereas the continuous data are presented as the mean \pm standard deviation (SD). A Chi-square test was used to evaluate the allelic and genotypic frequencies calculated from the observed genotypic counts. The Chi-square test was used for categorical variables and the unpaired Student $t$-test for continuous variables; the baseline characteristics were compared between those with or without hypertension and with or without the -395A allele. A previous study reported a significant association between the G-395A polymorphism and hypertension in females and nonsmokers (Wang et al., 2010); therefore, we performed subgroup analyses based on the gender and smoking status. Odd's ratio (OR) and 95\% confidential intervals $(\mathrm{Cl})$ were determined for hypertension using binary logistic regression models. All statistical analyses were performed using the SPSS for Windows software package (v.11.5; SPSS Inc, Chicago, IL, USA). A two-tailed P $<0.05$ was considered to be statistically significant.

\section{RESULTS}

\section{Characteristics of the study population}

In this study, 160 participants were excluded because of the lack of DNA samples. Overall, 710 participants ( 224 men and 486 women) were included in the analyses. There were no significant differences between the included and excluded populations in terms of age (93.5 \pm 3.2 vs $93.2 \pm$ $2.8, \mathrm{P}=0.587$ ), gender (women: 68.5 vs $65.0 \%, \mathrm{P}=0.325$ ), or the percentage of hypertension (60.8 vs $62.5 \%, P=0.368)$. All participants were Han Chinese.

Table 1 summarizes the characteristics of the included subjects with or without hypertension. The BMI of the subjects in the hypertension group was significantly lower than that of people in the control group (18.6 vs 19.6, $\mathrm{P}<0.001)$. No significant difference was found with respect to age, gender, smoking, diabetes, TG, TC, HDL-C, and LDL-C between the hypertension and control groups.

\section{Genotype distribution of KLOTHO G-395A}

The genotype frequencies of the KLOTHO G-395A polymorphism in the study population were $1.7 \%$ for $A A(N=12), 29.0 \%$ for $G A(N=206)$, and $69.3 \%$ for $G G(N=492)$; this was in compliance with Hardy-Weinberg equilibrium $(P=0.069)$. The allele frequencies were 0.838 for the $\mathrm{G}$ allele and 0.162 for the A allele in the entire study population. Significant differences were identified in the genotype distribution of the G-395A polymorphism between the hypertension and control groups (Table 1). The allele frequencies were 0.863 for the $\mathrm{G}$ allele and 0.137 for the $\mathrm{A}$ allele in the hypertension group, whereas the corresponding frequencies were 0.800 and 0.200 , respectively, for the control group $(P<0.001)$. 


\begin{tabular}{|c|c|c|c|}
\hline & Control $(N=278)$ & Hypertension $(\mathrm{N}=432)$ & $P$ value \\
\hline Age (years) & $93.4 \pm 3.2$ & $93.7 \pm 3.3$ & 0.331 \\
\hline Female (\%) & 69.4 & 67.1 & 0.534 \\
\hline Smoker (\%) & 64.0 & 62.0 & 0.592 \\
\hline $\mathrm{BMI}\left(\mathrm{kg} / \mathrm{m}^{2}\right)$ & $19.6 \pm 3.8$ & $18.6 \pm 3.3$ & $<0.001$ \\
\hline Diabetes (\%) & 8.3 & 5.6 & 0.155 \\
\hline $\mathrm{TG}(\mathrm{mM})$ & $4.9 \pm 1.6$ & $4.0 \pm 0.8$ & 0.348 \\
\hline $\mathrm{TC}(\mathrm{mM})$ & $1.3 \pm 0.8$ & $1.2 \pm 0.5$ & 0.132 \\
\hline HDL-C (mM) & $1.6 \pm 0.8$ & $1.6 \pm 0.6$ & 0.425 \\
\hline LDL-C (mM) & $2.3 \pm 0.6$ & $2.2 \pm 0.6$ & 0.075 \\
\hline \multicolumn{4}{|c|}{ Genotype distribution } \\
\hline GG (\%) & 64.0 & 72.7 & $<0.001$ \\
\hline GA (\%) & 32.0 & 27.1 & \\
\hline AA (\%) & 4.0 & 0.2 & \\
\hline
\end{tabular}

All data represents the mean \pm standard deviation $(\mathrm{SD})$ unless otherwise indicated. $\mathrm{BMI}=$ body mass index; $\mathrm{HDL}-\mathrm{C}=$ high density lipoprotein cholesterol; LDL-C = low density lipoprotein cholesterol; TC = total cholesterol; TG = triglycerides.

In order to compare the different genotypes with G-395A polymorphism, the subjects were divided by $A$ allele into $A$ allele carriers versus non-carriers (GA+AA vs $G G$ ). Table 2 summarizes the characteristics of the subjects included in the $G G$ and $G A+A A$ genotype groups. The mean SBP was significantly higher in the GG group than in the GA+AA genotype group; however, the DBP did not differ significantly between the two groups. The percentages of hypertension and ISH were significantly higher in the GG genotype group than in the GA+AA genotype group. In addition, no significant differences were found between the two groups with respect to age, gender, smoking status, BMI, diabetes, TG, TC, HDL-C, and LDL-C.

Subgroup analyses revealed that the genotype distribution of the G-395A polymorphism between the hypertension and control groups were significantly different in women and smokers, but not in men or non-smokers (Table 3).

Table 2. Characteristics of study subjects with respect to the G-395A polymorphism in the KLOTHO gene.

\begin{tabular}{lccr}
\hline & GG $(\mathrm{N}=492)$ & GA+AA (N = 218) & P value \\
\hline Age (years) & $93.6 \pm 3.3$ & $93.3 \pm 3.0$ & 0.241 \\
Female (\%) & 67.3 & 72.1 & 0.312 \\
Smoker (\%) & 63.4 & 61.5 & 0.621 \\
SBP (mmHg) & $143.6 \pm 24.2$ & $133.9 \pm 19.7$ & $<0.001$ \\
DBP (mmHg) & $73.0 \pm 12.1$ & $71.9 \pm 12.0$ & 0.242 \\
Hypertension (\%) & 63.8 & 54.1 & 0.015 \\
ISH (\%) & 47.0 & 38.1 & 0.029 \\
BMI (kg/m) & $19.5 \pm 3.4$ & $19.1 \pm 3.7$ & 0.155 \\
Diabetes (\%) & 7.9 & 6.1 & 0.401 \\
TG (mM) & $4.8 \pm 3.7$ & $4.1 \pm 0.8$ & 0.493 \\
TC (mM) & $1.3 \pm 0.8$ & $1.2 \pm 0.5$ & 0.327 \\
HDL-C (mM) & $1.6 \pm 0.8$ & $1.6 \pm 0.7$ & 0.503 \\
LDL-C (mM) & $2.3 \pm 0.5$ & $2.2 \pm 0.6$ & 0.675 \\
\hline All & & & \\
\hline
\end{tabular}

All data represents the mean \pm SD unless otherwise indicated. BMI = body mass index; $\mathrm{DBP}=$ diastolic blood pressure; HDL-C = high density lipoprotein cholesterol; ISH = isolated systolic hypertension; LDL-C = low density lipoprotein cholesterol; SBP = systolic blood pressure; TC = total cholesterol; TG = triglycerides.

\section{Association between hypertension and the KLOTHO G-395A polymorphism}

Table 4 shows the results of unadjusted and adjusted logistic regression models regarding the association between hypertension and the KLOTHO G-395A gene polymorphism. Compared 
to subjects with the GG genotype, subjects with the GA+AA genotype showed a significantly lower risk of hypertension even after adjusting for age, gender, and other relevant risk factors (OR 0.68, $95 \% \mathrm{Cl}=0.49$ to 0.95$)$.

Table 3. Subgroup analysis of the association between KLOTHO G-395A genotypes and hypertension with respect to the gender and smoking status.

\begin{tabular}{|c|c|c|c|c|}
\hline & & Hypertension & Control & $P$ value \\
\hline \multicolumn{5}{|l|}{ Gender } \\
\hline \multirow[t]{2}{*}{ Men $(N=224)$} & GG (\%) & 75.2 & 67.0 & 0.182 \\
\hline & $\mathrm{GA}+\mathrm{AA}(\%)$ & 24.8 & 33.0 & \\
\hline \multirow[t]{2}{*}{ Women $(N=486)$} & GG (\%) & 71.6 & 62.6 & 0.038 \\
\hline & $\mathrm{GA}+\mathrm{AA}(\%)$ & 28.4 & 37.4 & \\
\hline \multicolumn{5}{|l|}{ Smoking status } \\
\hline \multirow[t]{2}{*}{ Smoker $(N=304)$} & GG (\%) & 73.5 & 64.6 & 0.045 \\
\hline & $\mathrm{GA}+\mathrm{AA}(\%)$ & 26.5 & 35.4 & \\
\hline \multirow[t]{2}{*}{ Nonsmoker $(N=401)$} & GG (\%) & 71.3 & 63.0 & 0.158 \\
\hline & GA+AA (\%) & 28.7 & 37.0 & \\
\hline
\end{tabular}

Table 4. Estimate of the effect of the G-395A polymorphism on hypertension modeled via logistic regression.

\begin{tabular}{lcc}
\hline & GG OR $(95 \% \mathrm{Cl})$ & $\mathrm{GA}+\mathrm{AA} \mathrm{OR}(95 \% \mathrm{Cl})$ \\
\hline Unadjusted & 1 (Reference) & $0.67(0.48,0.93)$ \\
Adjusted model $1^{\dagger}$ & 1 (Reference) & $0.66(0.48,0.91)$ \\
Adjusted model $2^{\ddagger}$ & 1 (Reference) & $0.68(0.49,0.95)$ \\
\hline
\end{tabular}

${ }^{\dagger}$ Adjusted for age and gender. ${ }^{\ddagger}$ Adjusted for age, gender, BMI, smoking, diabetes, use of anti-hypertensive drugs, TG, $\mathrm{TC}, \mathrm{HDL}-\mathrm{C}$, and LDL-C. BMI = body mass index; $\mathrm{Cl}=$ confidence interval; $\mathrm{HDL}-\mathrm{C}=$ high density lipoprotein cholesterol; LDL-C = low density lipoprotein cholesterol; OR = odd's ratio; TC = total cholesterol; TG = triglycerides.

\section{DISCUSSION}

The results of our study indicated that the A allele carriers of the KLOTHO G-395A SNP were significantly less common in the hypertension group than in the control group. The KLOTHO G-395A SNP was associated with hypertension after adjusting for age, gender, and other relevant risk factors. These findings confirmed the results of a previous study that was also conducted in the Chinese Han population (Wang et al., 2010).

In our study, the frequency ofexpression of the A allele in theKLOTHO G-395A SNP was 0.162. In previous studies, the frequencies of the A allele in the G-395A SNP varied from 0.155 (Kim et al., 2008) to 0.171 (Rhee et al., 2006c) in Korean populations, 0.128 (Kawano et al., 2002) to 0.168 (Shimoyama et al., 2009) in Japanese populations, 0.191 in a Chinese Han population (Wang et al., 2010), and 0.227 in a Turkish population (Telci et al., 2011). Our results on the allele frequency were similar to those of studies conducted in East Asian populations, but not to that seen in the study conducted in the Turkish population. This finding suggests that the KLOTHO G-395A SNP may vary in different ethnic populations.

In this study, the mean SBP was significantly higher in the GG genotype group than in the GA+AA group; however, this finding was inconsistent with the results of previous studies (Rhee et al., 2006b; Shimoyama et al., 2009). Rhee et al. (2006b) found that the mean SBP was higher in -395A allele carriers compared to the non-carriers in healthy Korean women (Rhee et al., 2006b), whereas Shimoyama et al. (2009) reported no significant difference in the mean SBP between 
-395A carriers and non-carriers in a healthy Japanese population. Notably, the two previous studies were both conducted in healthy populations, and the differences may be attributed to the lack of hypertension patients in the study population. Another possibility could be the differences in genetic backgrounds between different ethnic populations. Wang et al. (2010) did not report this outcome in a Chinese population; therefore, we could not compare their results with ours. However, the comparable difference in the association between KLOTHO G-395A SNP and coronary heart disease may support this hypothesis. For example, the -395 A allele of the KLOTHO gene was considered to be a protective factor for coronary heart disease in a Korean population (Rhee et al., 2006a), but a risk factor in a Japanese population (Imamura et al., 2006).

The reason for this difference in the association between the KLOTHO G-395A SNP and hypertension between men and women remains unclear. However, this finding was consistent with that of a previous study, which discovered significant associations between the G-395A SNP and essential hypertension in women, but not in men (Wang et al., 2010). One possibility is the genderbased difference in the maintenance of free radical homeostasis (Ali et al., 2006). The KLOTHO gene reduces oxidative stress by inhibiting the insulin/IGF-1 signaling pathway (Wang and Sun, 2009); however, oxidative stress may play a role in the genesis of hypertension (Harrison and Gongora, 2009).

In this study, the KLOTHO G-395 SNP was significantly associated with hypertension in smokers; however, this was inconsistent with the results obtained by Wang et al. (2010), who found that the corresponding association was significant in nonsmokers. This difference might be attributed in part to the different definitions of smokers. In this study, smokers were defined as subjects who smoked $\geq 100$ cigarettes in their lifetime, according to the National Health Interview Survey (NHIS) (Ryan et al., 2012) definition of a smoker. Alternately, Wang et al. (2010), defined smokers as subjects who smoked $\geq 20$ cigarettes/day for $>2$ years. In addition, the subjects included in our study had an extremely high rate of smoking $(62.8 \%)$, because of the tradition of smoking self-made cigarettes ("Chinese-prepared tobacco") in the Dujiangyan area.

The molecular mechanism with which the KLOTHO G-395A SNP modulated the blood pressure remains unclear. Animal studies indicated that the KLOTHO gene could protect against endothelial dysfunction (Nzietchueng et al., 2011). Yang et al. (2003) reported that the KLOTHO gene might play a role in the regulation of vascular tone through a homeostatic interplay between $\mathrm{NO}$ and the renin-angiotensin system. This might explain the association between the KLOTHO gene and hypertension. In addition, KLOTHO gene delivery is known to ameliorate vascular endothelial function and reduce blood pressure in rat models of atherosclerotic disease (Saito et al., 2000). Recently, Wang et al. (2010) reported that the -395A variant of the G-395A SNP might protect against the presence and development of essential hypertension by upregulating KLOTHO gene expression and increasing KLOTHO protein levels. This was believed to increase the NO production, alleviate arteriosclerosis, and suppress oxidative stress. However, further studies are required to investigate the complex regulatory mechanisms.

There are some limitations to this study. Firstly, 160 subjects were excluded from this study because of a lack of DNA sample availability. The exclusion of participants with missing data may undermine the association between the KLOTHO G-395A SNP and hypertension. Secondly, there was a gender imbalance in the study population, which is a common characteristic of an oldest-old population because women generally have a longer expected lifespan than men. Thirdly, approximately $15 \%$ of the hypertension patients in our study population used anti-hypertensive agents during the study, which may have influenced the blood pressure test results. Therefore, 
the percentage of ISH patients might have been underestimated. Lastly, all participants were $\geq 90$ years of age; in other words, all of the study participants were survivors of the whole population. This may induce selection bias in our study.

In conclusion, the KLOTHO G-395A SNP is associated with hypertension in Chinese nonagenarians and centenarians. The $-395 \mathrm{~A}$ allele of the KLOTHO gene may be a protective genetic factor for hypertension in the Chinese population. Further studies with a larger sample size are required to confirm this finding, and to identify the possible mechanism with which KLOTHO G-395A influences the blood pressure.

\section{Conflicts of interest}

The authors declare no conflict of interest.

\section{ACKNOWLEDGMENTS}

Research supported by the project of Science and Technology Bureau of Sichuan Province (\#2006Z09-006-4) and the Cadres Health Care Committee of the Sichuan Province Health Department (\#2012-GANJIANBAN-7). The authors would like to acknowledge the staff of the Department of Geriatrics, Dujiangyan Hospital, and all the participants of the study and their legal proxies, for their invaluable contributions to the study.

\section{REFERENCES}

Ali SS, Xiong C, Lucero J, Behrens MM, et al. (2006). Gender differences in free radical homeostasis during aging: shorter-lived female C57BL6 mice have increased oxidative stress. Aging Cell 5: 565-574.

Chobanian, AV, Bakris GL, Black HR, Cushman WC, et al. (2003). Seventh report of the Joint National Committee on the prevention, detection, evaluation, and treatment of high blood pressure. Hypertension 42: 1206-1252.

Delles C, McBride MW, Graham D, Padmanabhan S, et al. (2010). Genetics of hypertension: from experimental animals to humans. Biochim. Biophys. Acta 1802: 1299-1308.

El Shamieh S, Herbeth B, Azimi-Nezhad M, Benachour H, et al. (2012). Human formyl peptide receptor 1 C32T SNP interacts with age and is associated with blood pressure levels. Clin. Chim. Acta 413: 34-38.

Expert Committee on the Diagnosis Classification of Diabetes Mellitus (2003). Report of the expert committee on the diagnosis and classification of diabetes mellitus. Diabetes Care 26 (Suppl 1): S5-S20.

Harrison DG and Gongora MC (2009). Oxidative stress and hypertension. The Medical clinics of North America 93: 621-635.

Imamura A, Okumura K, Ogawa Y, Murakami R, et al. (2006). Klotho gene polymorphism may be a genetic risk factor for atherosclerotic coronary artery disease but not for vasospastic angina in Japanese. Clin. Chim. Acta 371: 66-70.

Kawano K, Ogata N, Chiano M, Molloy H, et al. (2002). Klotho gene polymorphisms associated with bone density of aged postmenopausal women. J. Bone Miner. Res. 17: 1744-1751.

Kim Y, Jeong SJ, Lee HS, Kim EJ, et al. (2008). Polymorphism in the promoter region of the klotho gene (G-395A) is associated with early dysfunction in vascular access in hemodialysis patients. Korean J Intern. Med. 23: 201-207.

Kim Y, Kim JH, Nam YJ, Kong M, et al. (2006). Klotho is a genetic risk factor for ischemic stroke caused by cardioembolism in Korean females. Neurosci. Lett. 407: 189-194.

Kunes $\mathrm{J}$ and Zicha J (2009). The interaction of genetic and environmental factors in the etiology of hypertension. Physiol. Res. 58: S33-S41.

Kuro-o M (2009). Klotho and aging. Biochim. Biophys. Acta 1790: 1049-1058.

Kuro-o M, Hanaoka K, Hiroi Y, Noguchi T, et al. (1995). Salt-sensitive hypertension in transgenic mice overexpressing $\mathrm{Na}(+)-$ proton exchanger. Circ. Res. 76: 148-153.

Nzietchueng R, el Shamieh S, Benachour H, Labat C, et al. (2011). Klotho KL-VS genotype is involved in blood pressure regulation. Clin. Chim. Acta 412: 1773-1777.

Rhee EJ, Oh KW, Lee WY, Kim SY, et al. (2006a). The differential effects of age on the association of KLOTHO gene polymorphisms with coronary artery disease. Metabolism 55: 1344-1351. 
Rhee EJ, Oh KW, Yun EJ, Jung CH, et al. (2006b). Relationship between polymorphisms G395A in promoter and C1818T in exon 4 of the KLOTHO gene with glucose metabolism and cardiovascular risk factors in Korean women. J. Endocrinol. Invest. 29: 613-618.

Ryan H, Trosclair A and Gfroerer J (2012). Adult current smoking: differences in definitions and prevalence estimates-NHIS and NSDUH, 2008. J. Environ. Public Health 2012: 918368.

Saito Y, Nakamura T, Ohyama Y, Suzuki T, et al. (2000). In vivo klotho gene delivery protects against endothelial dysfunction in multiple risk factor syndrome. Biochem. Biophys. Res. Commun. 276: 767-772.

Shimoyama Y, Nishio K, Hamajima N and Niwa T (2009). KLOTHO gene polymorphisms G-395A and C1818T are associated with lipid and glucose metabolism, bone mineral density and systolic blood pressure in Japanese healthy subjects. Clin. Chim. Acta 406: 134-138.

Telci D, Dogan AU, Ozbek E, Polat EC, et al. (2011). KLOTHO gene polymorphism of G395A is associated with kidney stones. Am. J. Nephrol. 33: 337-343.

Virdis A, Bruno RM, Neves MF, Bernini G, et al. (2011). Hypertension in the elderly: an evidence-based review. Curr. Pharm. Des. 17: 3020-3031.

Wang HL, Xu Q, Wang Z, Zhang YH, et al. (2010). A potential regulatory single nucleotide polymorphism in the promoter of the Klotho gene may be associated with essential hypertension in the Chinese Han population. Clin. Chim. Acta 411: 386-390.

Wang Y and Sun Z (2009). Current understanding of klotho. Ageing Res. Rev. 8: 43-51.

Yang J, Matsukawa N, Rakugi H, Imai M, et al. (2003). Upregulation of cAMP is a new functional signal pathway of Klotho in endothelial cells. Biochem. Biophys. Res. Commun. 301: 424-429.

Yue JR, Dong BR, Huang CQ, Lu ZC, et al. (2010). Pro12Ala polymorphism in PPAR-gamma2 and dementia in Chinese nonagenarians/centenarians. Age 32: 397-404.

Zeglin MA, Pacos J and Bisognano JD (2009). Hypertension in the very elderly: Brief review of management. Cardiol. J. 16: 379-385.

Zhou Y, Huang CQ, Lu ZC and Dong BR (2012). Association of serum uric acid with Pro12Ala polymorphism in PPAR-gamma2 among Chinese nonagenarians/centenarians. Aging Clin. Exp. Res. 24: 139-144. 